\title{
Wasting away
}

A leading cause of death in infants is spinal muscular atrophy (SMA). This genetic disorder arises from mutation of the telomeric copy (SMN1) of a duplicated gene. Although some full-length SMN protein is produced from the centromeric gene (SMN2) in affected individuals, the level of this protein is insufficient to prevent lethality in motor neurons.

SMN protein is found in the cytoplasm, where it associates with the Sm snRNP core protein, and in nuclear Cajal bodies/gems, where it associates with Gemins 2, 3 and 4. Previously, SMN was shown to participate in snRNP assembly in the cytoplasm and in splicing of premessenger RNA in the nucleus. Some defects in SMN prevent its oligomerization, which is a prerequisite for the protein's interaction with $\mathrm{Sm}$ protein and Gemin 3.

Recently, Dreyfuss et al. (J. Cell Biol. 152, 75-85; 2000) defined a new functional interaction that links SMN and RNA polymerase II (RNAP II). Using nanoelectrospray mass spectrometry, and immunoprecipitation and in vitro-binding assays, they identified RNA helicase A (RHA) as another SMN-interacting protein. Point mutation or carboxy-terminal truncation of SMN rendered it unable to bind to RHA and a mutant protein lacking the amino-terminal 27 amino acids (SMN $\Delta$ N27), although able to bind to RHA, acted in a dominant negative manner. The RGG-rich C-terminal domain (CTD) of RHA mediates its interaction with SMN. Furthermore, SMN interacts with the CTD of RNAP II, and this association is enhanced when RHA is present. However, these studies did not determine whether RHA forms a bridge between SMN and the CTD of RNAP II, or whether it stabilizes a direct association between them.

Transfection of SMN $\Delta$ N27, but not wild-type SMN, led to mislocalization of RNAP lla (along with other components of the transcriptional machinery, such as RHA) from a punctate nucleoplasmic distribution to an accumulation in Cajal bodies/gems, whereas RNAP Ilo relo-

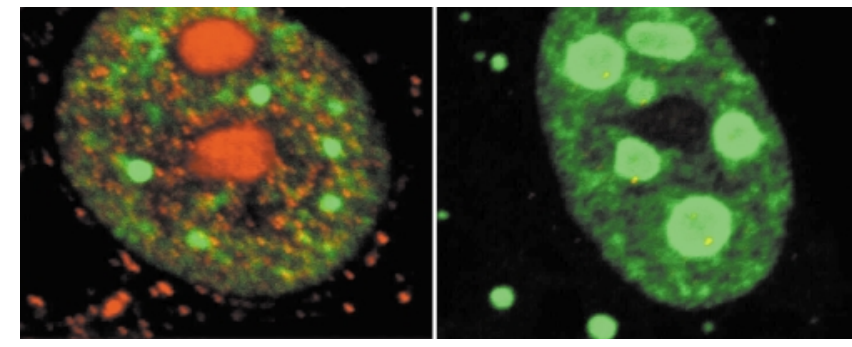

IMAGE COURTESY OF ROCKEFELLER UNIVERSITY PRESS

calized from the nucleoplasm to interchromatin granule clusters surrounding Cajal bodies/gems. In the cytoplasm, transfection of SMN $\Delta$ N27 protein resulted in aggregation of Sm proteins. However, the localization of hnRNP and SR proteins was not affected when the mutant SMN protein was present.

Given these differences, it was interesting to discover whether mislocalization of these proteins affected transcription itself. When wildtype SMN was transfected into cells, a high level of transcription was observed in both the nucleoplasm and nucleolus (left picture; SMN protein in green, RNA transcription shown in red). When SMN $\Delta$ N27 was used, however, transcription in both domains was inhibited (right picture), indicating that both RNAP II and RNAP I were affected. Therefore, some defective SMN proteins, such as SMN $\Delta$ N27, can affect transcription because they sequester RNA polymerase inapropriately and prevent active complexes from forming, whereas other mutant SMN proteins prevent any interaction with RNA polymerase. These results lead to a working model in which SMN protein may be an integral factor that directs transcriptosome assembly.

ANGELA EGGLESTON 